\title{
Throughput Validation of an Advanced Channel Assignment Algorithm in IEEE 802.11 WLAN
}

\author{
Mohamad Haidar \\ Department of Electrical \\ Engineering \\ Ecole de Technologie Superieure \\ Montreal, QC, H3C 1K3, Canada \\ Email: \\ Mohamad.Haidar.1@ens.etsmtl.ca \\ Hussain Al-Rizzo, Yupo Chan \\ Systems Engineering \\ Department \\ University of Arkansas at Little \\ Rock \\ Little Rock, AR, 72204, USA \\ Email: \{hmalrizzo, \\ yxchan\}@ualr.edu
}

\begin{tabular}{ll}
\multicolumn{1}{c}{ Robert Akl } & \multicolumn{1}{c}{ Mohamad Bouharras } \\
Department of Computer & Department of Electrical \\
Science and Engineering & Engineering \\
University of North Texas & Ecole de Technologie \\
Denton, TX, 76203, USA & Superieure \\
Email: rak1@cse.unt.edu & Montreal, QC, H2J 3A6, \\
& Canada \\
& Email: \\
& mbouharras@ gmail.com
\end{tabular}

\begin{abstract}
In this paper, an enhanced channel-assignment algorithm at the Access Points (APs) of a Wireless Local Area Network (WLAN) is validated. The algorithm aims to maximize the Signal-to-Interference Ratio (SIR) at the user level in order to determine the appropriate channel at the APs. The initial channel assignment step at the APs is based on minimizing the total interference between APs. Based on this initial assignment, the SIR for each user is calculated. Then, another channel assignment is performed based on maximizing the SIR at the users. The algorithm can be applied to any WLAN, irrespective of the user distribution and user load. Results show that the algorithm is capable of significantly increasing the SIR over the WLAN, which in turn should improve throughput. Therefore, we use OPNET simulation tool to construct several realistic scenarios in order to validate our results.
\end{abstract}

Index Terms-Signal-to-Interference Ratio; WLAN; Channel Assignment; Access Points; OPNET.

\section{INTRODUCTION}

The frequency spectrum available for WLAN operations in North America is limited to eleven frequency channels in the $2.4 \mathrm{GHz}$ band out of which three are non-overlapping and are allocated for $802.11 \mathrm{~b}$ and $802.11 \mathrm{~g}$ operations [1]. Due to the limited number of WLAN operating channels available, frequency channels need to be carefully assigned to APs so that the resulting network can maintain an adequate SIR level.

Channel assignment in IEEE 802.11 WLAN has received significant attention in the past few years [2]-[7]. The increase in deployment of APs has led researchers to develop channel assignment algorithms in order to reduce co-channel and adjacent channel interferences from neighboring APs, which causes an overall degradation in the throughput of the network.

The authors in [2] noted that previous AP placement and channel assignment were always designed sequentially. Therefore, they proposed an integrated model that addresses both at the same time. They showed that, by combining AP placement and channel assignment, the results were superior. In [3], the authors proposed an approach for optimizing channel assignment for hot-spot service areas in a WLAN by formulating an Integer Linear Programming (ILP) model. Their objective was to minimize the maximum channel utilization, thus equalizing the load distribution. This results in a higher throughput, through assigning non-overlapping channels among neighboring APs. A dynamic channelassignment ILP that minimizes channel interference between neighboring APs at a reference AP was presented in [4]. The channel assignment was done at the initial phase of planning, setting aside user considerations. The authors in [5] applied the concept of channel assignment in the outdoor environment to the indoor environment. They installed three IEEE 802.11 compliant APs in an indoor environment and performed signal measurements to assign channels for the APs. Then, an ILP assigns channels to APs. The authors in [6] proposed a weighted variant of the coloring graph algorithm to improve the usage of wireless spectrum in WLANs. The authors emphasized that a least congested channel assignment is not efficient with the continued growth of WLANs. Finally, we presented a channel assignment algorithm in [7] that minimizes the interference between neighboring APs after achieving a load-balanced state based on our work published in [8]. Our algorithm showed great improvement in results when channel assignment was applied after load balancing was achieved on the WLAN. In [9], we developed a mathematical model for the channel assignment algorithm based on SIR at the user level. The algorithm provided better results compared to previous work where channel assignment was made at an initial stage with no considerations given to users.

In this paper, we extend our work presented in [7], [8], and [9] by constructing several scenarios using OPNET simulation tool to validate that such an improvement of SIR at the user level leads to a better throughput at the user as well as on the network. However, some of the results previously published [9] are provided in this paper for the purpose of comparison with the constructed scenarios in OPNET. Therefore, a brief description of the channel assignment algorithm based on SIR at the user level is followed for clarity reasons. The channel assignment algorithm is performed in two steps: An initial channel assignment is conducted based on [7], where channels are assigned to APs after a loadbalanced state is achieved [8], and then SIR is computed at each user to reassign channels to APs based on maximizing 
the SIR in the second step [9]. The algorithm in [8] deals with distributing the load more efficiently among APs by reassigning users to different APs while decrementing the transmitted power of the Most Congested AP (MCAP) in discrete steps. The current paper goes one step further to reassign channels based on maximizing the SIR at the users and validates the analytical results through various OPNET [10] simulation scenarios.

Related work has considered minimizing the interference between neighboring APs. This could be an efficient channel assignment approach for small-scale WLANs where users are not largely populated in the service areas. However, as the users populate the network, a more suitable channel assignment based on the users' needs is required. In reality users distribute themselves at different locations in the study area. It should be emphasized that, to best of the authors' knowledge, the current paper is the first attempt to consider assigning channels to APs based on maximizing the SIR at the user level, which quantitatively leads to increase in network throughput [11].

The remainder of this paper is organized as follows: In section II, we define our channel assignment model and algorithm. Numerical results are presented in section III. In section IV, OPNET simulation scenarios are presented, and finally section $\mathrm{V}$ concludes the paper.

\section{THE CHANNEL ASSIGNMENT MODEL}

Here, a brief description of the frequency channelassignment model for IEEE 802.11 WLAN systems published in [9] is presented. Frequency channels are assigned to each AP in such a way to maximize the SIR at the user level, rather than to minimize interference among APs. Maximizing the SIR of the whole users on the network leads to utilizing the channels (resources) more efficiently resulting in higher throughput [11]. Since channel resources are limited in IEEE $802.11 \mathrm{~b} / \mathrm{g}$, some frequency channels may need to be assigned to several APs. Assigning the same channel to two or more APs which are located far enough from each others is encouraged when the overlapping channel interference signal detected by each AP is be less than a given threshold.

A WLAN consisting of $M$ APs situated in a single-floor service area is considered. A set of randomly distributed $N$ users are to be served by these APs. Our algorithm is initiated by balancing the load based on power management algorithm in [8]. Each user is assigned to one and only one AP at any time. Under normal channel conditions, this user to AP assignment is assumed fixed throughout the simulation period.

The received power level for each user is evaluated using the No-Line-of-sight (NLOS) path-loss model in (1) [12].

$$
P L(d)=P L_{0}+29.4 * \log _{10}(d)+6.1 * x_{\alpha} \log _{10}(d)+24 y+1.3 * x_{s} y
$$

where $P L_{0}$ is the free space path loss, $d$ is the distance between user $i$ and $A P_{j}$, and $x_{a}, x_{s}$, and $y$ are mutually independent Gaussian random variables of zero mean and unit variance. These random variables model power loss due to factors other than distance.

The formulation of our channel-assignment problem as a Non-Linear Integer Programming (NLIP) model using the following variables is defined below [9]:

- $\quad \mathbf{A}_{\mathbf{j}}$ is the set of neighboring APs to $A P_{j}$.

- $K$ is the total number of available channels, 11 in IEEE $802.11 \mathrm{~b} / \mathrm{g}$.

- $\quad P_{i k}$ is the power received by user $i$ associated with $A P_{k}$.

- $\quad P_{i j}$ is the power received by user $i$ from the interfering $A P_{j}$.

- $\quad I_{i j}$ is the total interference experienced by user $i$ due to all APs $j$ (where $j \neq k$ ).

The channel assignment problem is modeled as the following [9]:

$$
\begin{gathered}
\max \sum_{i=1}^{N} \sum_{j=1}^{M} \operatorname{SIR}_{i j}(k), j \neq k \\
\text { subject to } \\
w_{j k}=\max \left(0,1-\left|C h_{j}-C h_{k}\right| \times c\right) \\
I_{i j}=\sum_{i=1}^{N} \sum_{j=1}^{M}\left(P_{i j} \bullet w_{j k}\right), \quad j \neq k \\
\operatorname{SIR}_{i j}(k)=\frac{P_{i k}}{I_{i j}} \quad \forall i, j, j \neq k
\end{gathered}
$$

$$
\begin{gathered}
j, k \in\{1, . ., M\} \\
i \in\{1, . . N\} \\
C h_{j}, C h_{k} \in\{1, . ., K\}
\end{gathered}
$$

Objective (2.1) maximizes the total SIR for all users $i$ associated with $A P_{k}$. Maximizing SIR is related to minimizing interference at the user level from neighboring APs which in turn, the interference at the user, is defined by the channel assignment. Constraint (2.2), which has been derived in our previous work [7], defines the interference overlap factor between $A P_{j}$ and $A P_{k}$, which have been assigned $C h_{j}$ and $C h_{k}$, respectively. Based on [7], the overlapping channel factor, $c$, is 0.2. Constraint (2.3) defines the interference experienced by user $i$ due to all neighboring APs except $A P_{k}$. Constraint (2.4) defines the signal-to-interference ratio for user $i$ due to interfering access points $j(j \neq k)$. The NLIP model determines the best integer variables $C h_{j}$ and $C h_{k}$ or channel assignments that lead to the maximum SIR among the whole users. This in turn results in the maximum throughput. It is observed that the non-linearity in the problem comes from the definition of the $w_{j k}$ variable, as shown in (2.2).

The following assumptions are considered when executed [9]:

- Each user $i$ updates his/her serving $\mathrm{AP}_{\mathrm{k}}$ with its associated $\operatorname{SIR}_{i}(k)=\sum_{j} \operatorname{SIR}_{i j}(k)$ upon registering with it;

- Each AP, synchronized with the other APs, will periodically request SIR from its associated users and transfer it to a central unit (Wireless/Wired server). In case of a change in the current user distribution, 
resulting from users joining or leaving the network, the $\operatorname{SIR}_{i j}(k)$ information at the central unit server runs the channel-assignment model to reassign channels to the APs.

- All APs are operated by the same internet service provider.

- $\quad$ The presented scenarios do not involve user mobility. User mobility could add a great value to the proposed algorithm that is why it is currently considered for future publications. Therefore, scenarios under consideration are set up with a fixed number of APs, a fixed number of users, and an average data rate over the study period.

- User-to-user interference was assumed negligible due to its low transmitted power compared to the AP's transmitted power.

- All users are continuously active.

- Data rate represents the average data rate over the simulation period (between $1 \mathrm{Mbps}$ and $6 \mathrm{Mbps}$ ) since it is very hard to capture data rate fluctuations.

- All simulations were run based on the IEEE 802.11g technology, i.e. 54 Mbps.

- APs transmitted power levels are set equally at $20 \mathrm{dBm}$ before power management algorithm in [8] is invoked.

- User sensitivity is set at $-90 \mathrm{dBm}$. In other words, any signal level stronger than the receiver sensitivity will be a potential association.

- The receiver detection threshold is assumed to be -110 $\mathrm{dBm}$. In other words, if the user is receiving a signal from an AP that falls below the detection threshold, then this signal is assumed to cause no interference at the receiver. Where as if the signal falls between receiver sensitivity and detection threshold that means the user causes interference.

The purpose of the displayed scenarios is to compare the effect of channel assignment at the initial design stage, in which the users have yet to enter the picture, and a later stage, when users are considered in the network.

The execution of the NLIP model can be described by a number of computational steps. Such a channel-assignment algorithm can be summarized as follows [9]:

1. Assign channels to the $M$ APs based on the NLIP model proposed in reference [7] which is based on minimizing the total interference between APs (initial stage).

2. Input the positions of $N$ randomly distributed users on the service area.

3. Perform load balancing based on the power-management algorithm proposed in [8].

4. The model in [8] provides the received power at each user.

5. Compute interference caused by neighboring APs at each user based on: First, distance between AP and user; Second, the interference overlap factor presented in [7] and equation (2.2).

6. Compute SIR for each user.

7. Run the NLIP model in equation (2).
The above algorithm is executed on a static environment i.e, at one time slice. If we were to assume continuity among time slices and that states transition smoothly from one time slice to another, then an additional step could be added to the channel assignment algorithm that involves repeating steps 2 7 in every new time slice. To test this hypothesis, a simulation is run continuously until the balanced load state discussed in [8] is achieved among data based on existing user patterns. Then, OPNET simulation tool was used to run real-time scenarios to affirm our simulation results. Because of the random distribution of the users, we ran more than 200 simulation replications for each scenario. It was judged that 200 replication cycles were sufficient to reach a steady state. During each replication cycle of the simulation, the association of user location $i$ to $\mathrm{AP}_{j}$ remains fixed-until a new association is obtained in step 3 . We show the average results of each scenario below followed by OPNET simulation results.

The authors solved the problem in equation (2) by enumeration using Matlab software tool rather than using an optimization solver. The reason behind using an enumeration method is to gain some insight on the SIR value for each iteration. SIR values were examined closely until a maximum was obtained. A more formal optimization routine that takes the computational complexity of the problem into consideration is currently considered for future publications.

\section{NUMERICAL RESULTS}

Several simulation scenarios were carried out with different WLAN service area sizes consisting of 4,6 , and 9 APs and 20,30, and 40 users, respectively. For complete set of results please refer to [9] for more details. In this section, we verify that the proposed channel assignment algorithm based on SIR at the user level does provide significant improvements at the network level in terms of delay and throughput than previous related art.

\section{A. Scenario Under Consideration}

The scenario under consideration is constructed in OPNET consisting of a grid of $2 \times 2$ APs and 20 uniformly distributed users over a $100 \mathrm{~m}$ x100 m area, Figure 1. APs are placed 60 meters away from each others. The objective is to compare the effect of the proposed channel assignment to other related work channel assignments at the user level. 


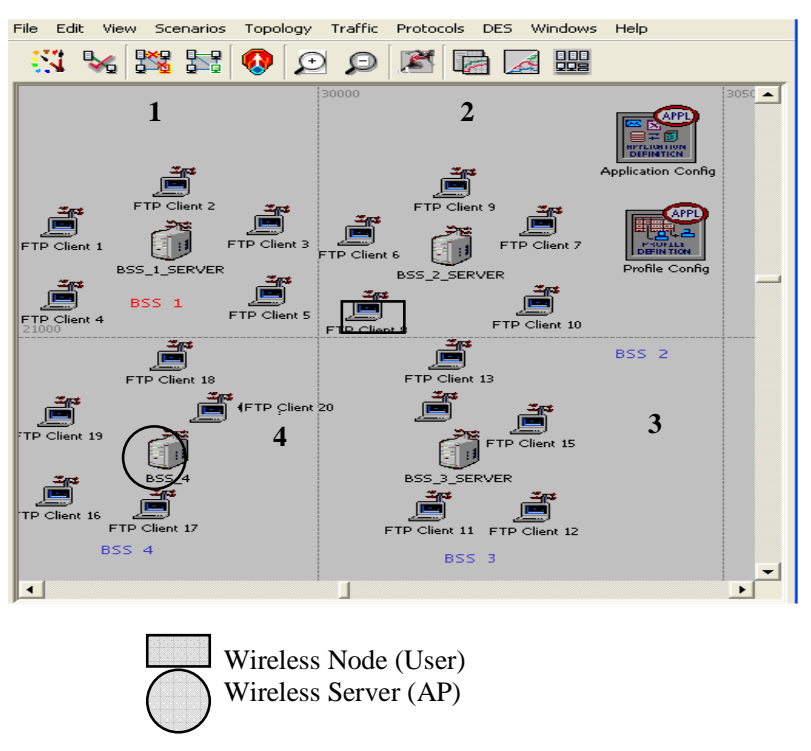

Fig. 1. 4 APs and 20 users WLAN in OPNET

The following assumptions were taken into consideration:

- All users are stationary.

- Power transmitted from each AP is $20 \mathrm{dBm}$.

- Receiver's threshold power is $-90 \mathrm{dBm}$.

- All APs' data rates are set to 11 Mbps.

- Each AP has 5 users that are uploading a 400 Kbytes file simultaneously to their respective wireless servers (APs).

- Simulation time is 1000 seconds (16 minutes and 40 seconds).

Four scenarios were conducted to study the effect of interference on the application level and the network level. In scenario 1 , channels $1,2,3$, and 4 were assigned to AP1 to AP4, respectively. In Scenario 2, non-overlapping channels 1, $6,1,11$ were assigned to AP1 to AP4, respectively, where the same channel, 1, was assigned to the diagonal APs. Optimal channel assignment, based on minimizing interference among neighboring APs, assigned 1, 8, 3, and 11, for AP1 to AP4, respectively in scenario 3 . Finally, channels $6,11,2$, and 1 , were assigned to AP1 to AP4 in scenario 4 based on maximizing the SIR at all user. One scenario was constructed and all other scenarios were duplicated while modifying the channels at each AP. Table I summarizes the channel assignment scenarios.

TABLE I- SUMMARY OF CHANNEL ASSIGNMENT SCENARIOS
\begin{tabular}{|c|c|c|c|c|}
\hline & $\begin{array}{c}\text { Scenario } \\
\mathbf{1}\end{array}$ & $\begin{array}{c}\text { Scenario } \\
\mathbf{2}\end{array}$ & $\begin{array}{c}\text { Scenario } \\
\mathbf{3}\end{array}$ & $\begin{array}{c}\text { Scenario } \\
\mathbf{4}\end{array}$ \\
\hline AP1 & 1 & 1 & 1 & 6 \\
\hline AP2 & 2 & 6 & 8 & 11 \\
\hline AP3 & 3 & 1 & 3 & 2 \\
\hline AP4 & 4 & 11 & 11 & 1 \\
\hline Color & Green & Red & $\begin{array}{c}\text { Dark } \\
\text { Blue }\end{array}$ & $\begin{array}{c}\text { Light } \\
\text { Blue }\end{array}$ \\
\hline
\end{tabular}

\section{B. Results}

Analysis of the different channel assignments is presented in this section. Results are intended to show the effect of channel assignment on the FTP upload response time at the application level and network level, as well as other network statistics.

Figure 2 shows comparison between the 4 channel assignment schemes in terms of global upload response time.

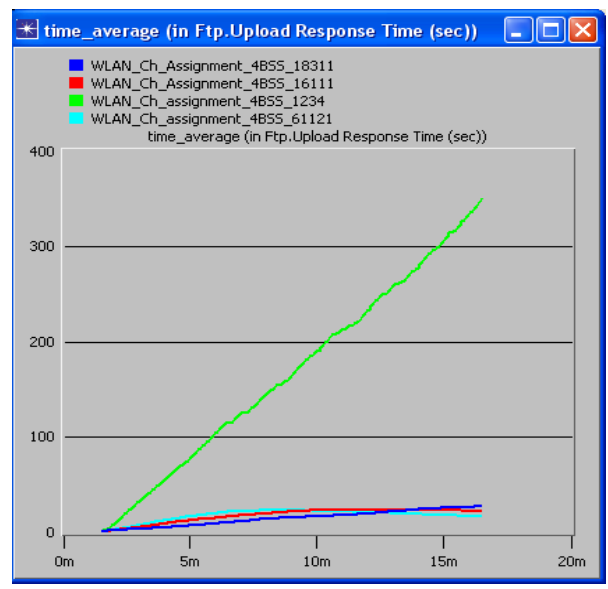

Figure 2. Global FTP upload response time (sec)

It is clear that the one-channel distance assignment causes the network's upload response time to increase linearly with simulation time. This is because all FTP clients associated with their respective AP are suffering from large interference from their neighboring APs which causes the Medium Access Control (MAC), of each FTP client, to continuously transmit packets but arriving to the intended AP with high bit error rates causing end to end delay. On the other hand, the remaining 3 channel assignment schemes perform very closely with the exception of scenario 4 (light blue), Figure 3, where response time after the $12^{\text {th }}$ minute starts falling below the other two competing channel assignments (red and dark blue). Therefore, in the long run, employing the channels assignment algorithm based on SIR provided better upload response time than the other approaches.

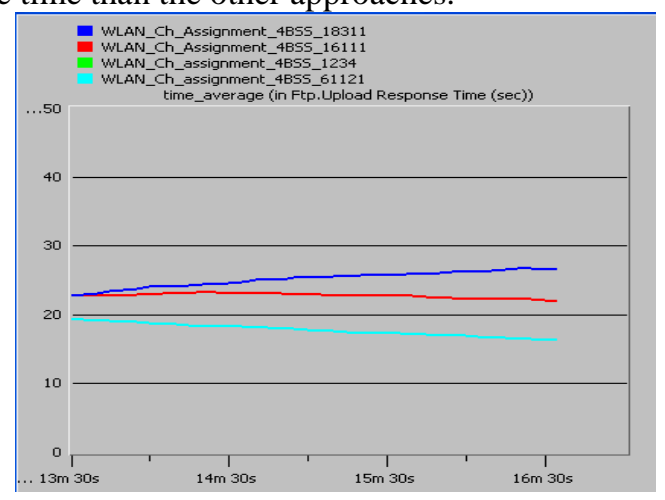

Figure 3. Zoomed in view of scenarios 2, 3, and 4. 
It is important to note that the channel assignment provided by scenario 4 is based on maximizing the SIR of the users. A different user distribution might lead a different channel assignment. Whereas, the channel assignment based on minimizing the interference between APs will remain the same as long as the AP distances are fixed.

Consequently, improving delay and response time of the network leads to a better network throughput, as shown in Figure 4.

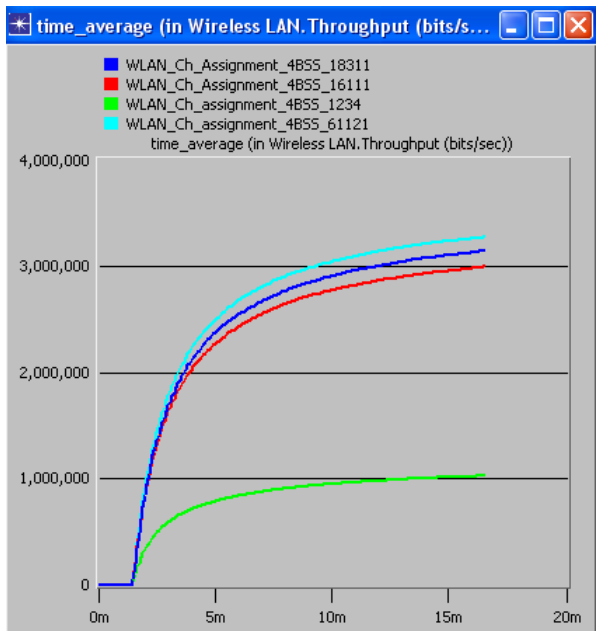

Figure 4. Global throughput of the 4-BSS WLAN

Furthermore, the delay at AP1, AP2, AP3, and AP4 are displayed in Figure 5, Figure 6, Figure 7, and Figure 8, respectively.

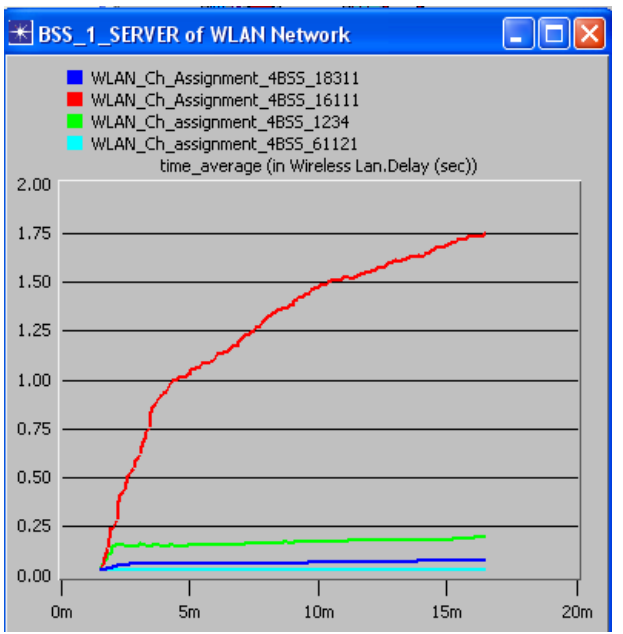

Figure 5. Delay at AP1 (sec)

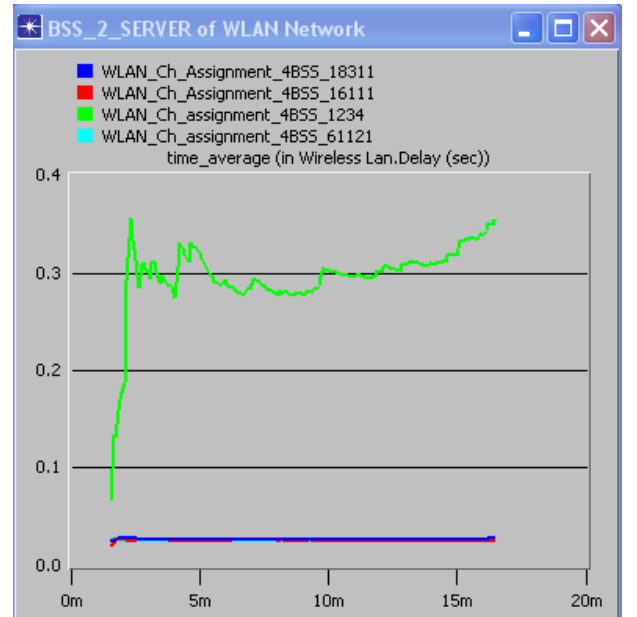

Figure 6. Delay at AP2 (sec)

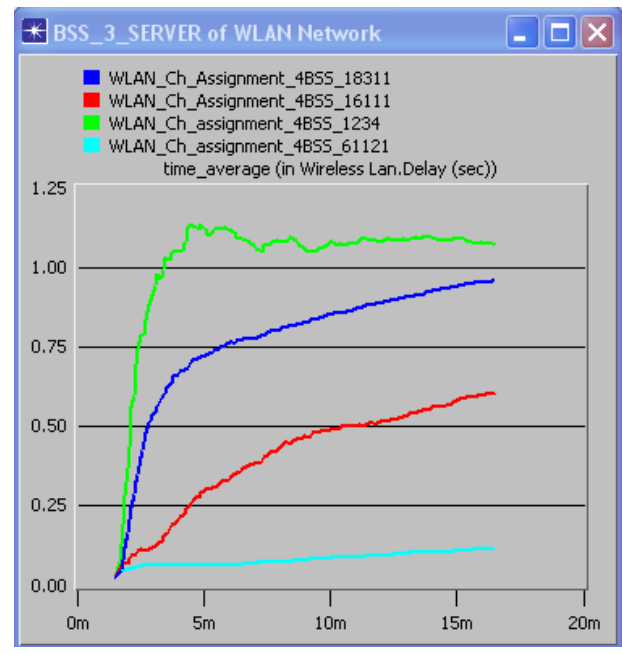

Figure 7. Delay at $\mathrm{AP} 3(\mathrm{sec})$

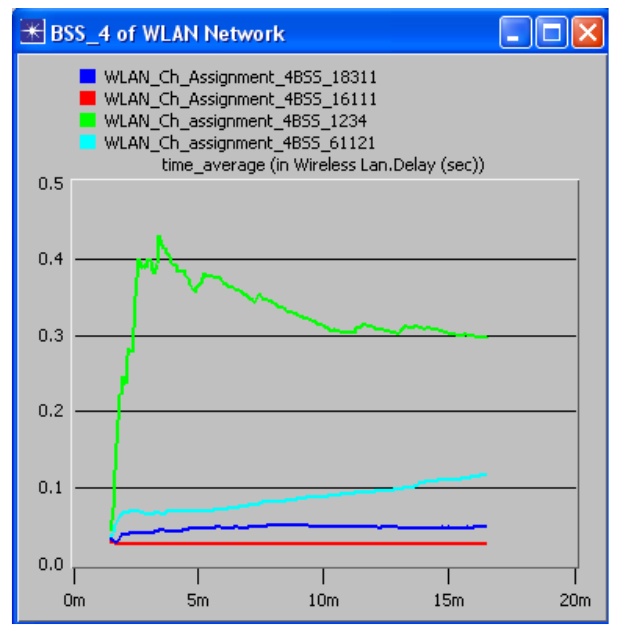

Figure 8. Delay at AP4 (sec)

It is noticed from the above figures that the onechannel distance scenario has the highest delay on AP2, AP3, and AP4. This is because the MAC transmits a packet and due to the high interference overlap in the channel assignment a collision takes place and the MAC has to defer transmission to 
another time interval, causing delay (after several collisions on the same packet). However, since AP1 and AP3 share the same channel " 1 " in scenario 2, the delay at AP1 from scenario 2 exceeds the other scenarios by far since collisions occur more frequently when the same channel.

As for the other three scenarios, it is determined that the channel assignment scenario based on SIR has less delay at AP1 and AP3 than the other two scenarios. This is because under this user distribution, the channels that lead to the maximum SIR are assigned. However, for AP2, it provides almost the same amount of delay as the other scenarios. Finally, the delay at AP4 is more than the other two algorithms. This can be explained by the fact that AP3 and AP4 have non-overlapping channels in the other competing scenarios: Scenario 2 (channel 3 and Channel 11 to AP3 and AP4, respectively) and scenario 3 (channel 1 and Channel 11 to AP3 and AP4, respectively). Therefore, it is expected to have more delay than the others, whereas, AP3 and AP4 are assigned channels 2 and 1, respectively, in scenario 4 leading to high interference on users.

In summary, the proposed channel assignment algorithm based on maximizing SIR, scenario 4, chooses the assignment of channels that leads to the best throughput on the network as shown in Figure 4.

\section{CONCLUSION}

In this paper, an advanced channel assignment algorithm based on maximizing the SIR at the users has been validated using OPNET. The algorithm extends the models presented in [7] and [8], where a channel assignment algorithm based on minimizing interference between neighboring APs was applied at the balanced load state, to include a channel reassignment at the balanced state by considering the SIR of the users. The algorithm has shown to provide better results compared to previous work where channel assignment was made at an initial stage with no considerations given to users, taking into consideration only interference between APs rather than SIR at the users. To support our findings, a real-time model was constructed in OPNET. Different channel assignment scenarios were implemented and results have shown the promised improvement in network throughput and delay if our algorithm is to be applied when users enter the network.

The problem discussed in this paper was developed for research development purposes and not for real-time applications, due to numerous existing complications. The model has proven to perform well for small networks. But due to the computational complexity of the problem defined in (2), future work could involve solving the NLIP by linearizing it. Interested researchers could be guided to a multicriteria optimization formulation after the linearization procedure is executed. This could lead to solving larger size networks efficiently. Upon solving the NLIP on a real time basis, one can include dynamic changes in the user's locations and mobility. In other words, the 7-step algorithm described in Section II would include optimizing over all instances when a user leaves or join a network. This would lead toward operational application of the NLIP model.

\section{ACKNOWLEDGMENT}

This research was funded by National Science Foundation EPSCoR (Grant number 0701890).

\section{REFERENCES}

[1]. "Supplement to IEEE Standard for Information Technology Telecommunications and Information Exchange between Systems Local and Metropolitan Area Networks - Specific Requirements. Part II: Wireless LAN Medium Access Control (MAC) and Physical Layer (PHY) Specifications: High-Speed Physical Layer Extension in the $2.4 \mathrm{GHz}$ Band," IEEE Standard 802.11b - 1999, pp. I - 90, 2000 .

[2]. Eisenblätter, A., Geerdes, H.-F. and Siomina, I., "Integrated Access Point Placement and Channel Assignment for Wireless LANs in an Indoor Office Environment," 8th IEEE Intl. Symposium on a World of Wireless, Mobile and Multimedia Networks, June 2007.

[3]. Y. Lee, K. Kim, and Y. Choi., "Optimization of AP placement and Channel Assignment in Wireless LANs" LCN 2002. 27 $7^{\text {th }}$ Annual IEEE Conference on Local Computer Networks, IEEE Computer Society, Washington D.C. USA, November 2002, pp. 831-836.

[4]. R. Akl and A. Arepally, "Dynamic Channel Assignment in IEEE 802.11 Networks," Proceedings of IEEE Portable 2007: International Conference on Portable Information Devices, March 2007

[5]. R. Rodrigues, G. Mateus, A. Loureiro, "Optimal Base Station Placement and Fixed Channel Assignment Applied to Wireless Local Area Network Projects," Seventh IEEE International Conference on Networks (ICON'99), 1999, pp.186.

[6]. Mishra, S. Banerjee, and W. Arbaugh, "Weighted Coloring Based Channel Assignment for WLANs," ACM SIGMOBILE Mobile Computing and Communications Review, vol.9, pp.19-31, 2005.

[7]. M. Haidar, H. M. Al-Rizzo, R. Akl, and Y. Chan," Channel Assignment and Load Distribution in a Power-managed WLAN," 18th Annual IEEE International Symposium on Personal, Indoor and Mobile Radio Communications, 3-7 September, 2007, Athens, Greece

[8]. M. Haidar, R. Akl, H. Al-Rizzo, Y. Chan, R. Adada, "Optimal Load Distribution in Large Scale WLAN Networks Utilizing a Power Management Algorithm," Proceedings of IEEE Sarnoff Symposium, May 2007.

[9]. M. Haidar, R. Ghimire, H. M. Al-Rizzo, R. Akl, and Y. Chan, "Channel Assignment in an IEEE 802.11 WLAN based on Signal-toInterference Ratio," 21st IEEE Canadian Conference on Electrical and Computer Engineering, May 4-7, 2008, Niagara Falls, ON, Canada.

[10].OPNET Technologies Inc., "OPNET," http://www.opnet.com [11].M. Boulmalf, H. El-Sayed, and A. Soufyane, "Measured Throughput and SNR of IEEE $802.11 \mathrm{~g}$ in a Small Enterprise Environment," $61^{\text {st }}$ Vehicular IEEE Vehicular Technology Conference, Vol. 2, pp. 1333-1337, Stockholm, Sweden, May 2005. [12]. J. Lei, R. Yates, L. Greenstein, and H. Liu, "Wireless Link SNR Mapping Onto An Indoor Testbed," Proceedings of IEEE Tridentcom 2005, pp.130-135, Trento, Italy, Feb. 2005. 